\title{
¿Sueñan los jueces con sentencias electrónicas?
}

\section{Do judges dream of electronic sentences?}

\author{
Raúl C. Cancio Fernández ${ }^{1}$
}

Fecha de recepción: 17/06/2020 - Fecha de aceptación: 23/07/2020

DOI : https://doi.org/10.22490/26655489.3854

Miembro del Gabinete Técnico del Tribunal Supremo de España

\section{Resumen}

Las voluntaristas esperanzas disruptivas en la plena aplicación de la inteligencia artificial (IA) en el ámbito del poder judicial no enerva una realidad ineludible -e inevitablecomo es la reformulada y creciente vinculación entre la IA y la Administración de Justicia. Y más concretamente, entre el algoritmo y la decisión judicial. Ahora bien, a lo largo del texto, se pondrá de manifiesto que, al día de hoy, la sustitución directa de la actividad humana en la decisión judicial es puramente quimérica a corto y medio plazo. Otra cosa son las actividades relacionadas con la predicción judicial al socaire de las plataformas privadas que emplean la IA, plenamente desarrolladas e implantadas (con algunas reticencias penales como en Francia). Junto a esta actividad comercial presciente, únicamente en dos ámbitos puede afirmarse con rigor una tímida disrupción en el ámbito judicial: las posibilidades que ofrece la automatización simple en el proceso y el empleo de tecnologías de procesamiento automático de datos en el ámbito audiovisual. Inconvenientes relacionados con el sesgo potencialmente discriminatorio; la pura búsqueda de la imitación tecnológica de las pautas de comportamiento humano; la desnaturalización del principio heurístico o incapacidad técnicas como el todavía incompleto procesamiento del lenguaje humano, son demasiadas servidumbres para no considerar aún más que una ficción a los jueces robots.

1 Letrado del Tribunal Supremo de España. Doctor en derecho y académico correspondiente de la Real Academia de Jurisprudencia y Legislación. 
Palabras clave: inteligencia artificial, decisión, sentencia, poder judicial, tribunales, disrupción, tecnología, algoritmo, sesgo, discriminación, privacidad, jueces.

\section{Abstract}

The voluntarist disruptive hopes in the full application of artificial intelligence (AI) in the field of the Judiciary does not enervate an inescapable - and inevitable- reality such as the reformulated and growing link between $\mathrm{Al}$ and the Administration of Justice. And more specifically, between the algorithm and the judicial decision. However, throughout the text, it will be shown that, to this day, the direct replacement of human activity in the judicial decision is purely chimerical in the short and medium term. Another thing is the activities related to the judicial prediction of the private platforms that use Al, fully developed and implemented (with some criminal reluctance as in France). Along with this prescient commercial activity, only in two areas can a timid disruption in the judicial field be rigorously affirmed: the possibilities offered by simple automation in the process and the use of automatic data processing technologies in the audiovisual field. Disadvantages related to potentially discriminatory bias; the pure search for the technological imitation of the guidelines of human behavior; the denaturation of the heuristic principle or technical incapacity, such as the still incomplete processing of human language, are too many servitudes to consider robot judges even more than a fiction.

Keywords: Artificial intelligence. Decision. Judgment. Judicial Branch. Courts. Disruption. Technology. Algorithm. Bias Discrimination. Privacy. Judges

¿Qué pasaría si supieras lo que tu juez querría escuchar? Context, Part of the Lexis Analytics ${ }^{\mathrm{TM}}$ Suite

Las voluntaristas esperanzas disruptivas en la plena aplicación de la inteligencia artificial (IA) en el ámbito del poder judicial no enerva una realidad ineludible -e inevitable- 
como es la reformulada y creciente vinculación entre la IA y la administración de justicia. Y más concretamente, entre el algoritmo y la decisión judicial.

Ahora bien, es conveniente, al hablar de estas cuestiones, hacerlo prevenido de ciertos adanismos perturbadores. La hogaño fascinación por la IA no se diferencia mucho de la que antaño experimentaron los ilustrados del siglo XVIII por el mecanicismo newtoniano, por los automaticismos como metáfora de la cultura manierista y reflejo de la idea que la Ilustración tiene del hombre, al que ve como una máquina, ya no regido por Dios sino por los propios mecanismos de sus vísceras, huesos y músculos. En este sentido, la aspiración de los revolucionarios ilustrados era configurar una administración a imagen y semejanza de una máquina que aplicara automáticamente la ley aprobada por el parlamento ${ }^{2}$.

Una fascinación, por tanto, de naturaleza onfaloscópica y nada original a la que hoy experimenta nuestra tecnificada sociedad por la robotización y las aplicaciones prácticas de la IA.

\section{El algoritmo y la decisión}

Los algoritmos, si atendemos al DRAE, son un conjunto ordenado y finito de operaciones que permite hallar la solución de un problema ${ }^{3}$. Etimológicamente, el origen del término se radica en el Bagdad del siglo IX, donde si bien no existían aún

2 "Por vez primera en la historia humana, todo el aparato del poder se objetiviza en un abstracto y casi mecánico (casi, porque enseguida habrá de aceptarse que la Ley puede y debe habilitar poderes llamados discrecionales en favor de las autoridades administrativas, poderes que facultan una posibilidad de elección entre soluciones igualmente justas) aparato de ejecución legal, de normas escritas y como tales ciertas, elaboradas meditadamente con la sage lenteur de las asambleas, fijas e inmutables para los intérpretes y para los aplicadores, seguras, pues, para los ciudadanos, que por vez primera en la historia pueden conocer el derecho a través de su publicación regular en su tenor exacto". García de Enterría, E: La lengua de los derechos. La formación del derecho público europeo tras la Revolución Francesa, Civitas, Madrid, 2009.

3 Una definición jurídica de algoritmo es ya posible encontrarla en diversos dictámenes de la Comisión de Garantía del derecho de acceso a la información pública de Cataluña. Así, la resolución de la reclamación 123/2016 señala que: "un algoritmo, como 'procedimiento de cálculo que consiste en cumplir un conjunto ordenado y finito de instrucciones con unos datos especificados para llegar a la solución del problema planteado' o 'conjunto finito de reglas que, aplicadas de manera ordenada, permiten la resolución sistemática de un problema, el cual se utiliza como punto de partida en programación informática" (en las dos definiciones que da el Termcat aplicables a este caso), no deja de ser un tipo de información, expresado habitualmente en lenguaje 
los ordenadores, sí impartía clases un brillante e inteligente matemático, Mohamed ben Musa, conocido como Al-khwarizmi, sobrenombre del que deriva el término alguarismo, ulteriormente contaminado por el latín medieval, que lo convirtió en algoritmo, merced a la palabra aritmo, número en griego.

La anfractuosidad de esta herramienta tecnológica se ha ido incrementando exponencialmente, pasando de tener una naturaleza estática a otra dinámica 0 , en otras palabras, si en el diseño de los primeros, los programadores insertaban en ellos los criterios para tomar las decisiones, en los algoritmos dinámicos, estos disponen ya de la capacidad de aprender con el tiempo de los datos y experiencias en aras de tomar decisiones autónomas, generando sus propias instrucciones desligadas por tanto de las pretéritamente aplicadas por el programador.

Un paso más en esta evolución lo constituiría el llamado aprendizaje profundo 0 deep learning que supone un funcionamiento de la IA emulando redes neuronales complejas. En estos casos, los algoritmos extraen patrones de ingentes masas de datos masivos caracterizados por su volumen, variedad y velocidad - las llamadas tres $\mathrm{V}$ - obteniendo resultados que ya no se vinculan de modo lineal sino complejo, por lo que no resulta en absoluto sencillo determinar la causalidad entre los datos manejados y la decisión finalmente adoptada. Esta evolución supone, en puridad, la huida de la decisión finalmente elegida del control del ser humano, incapaz de comprender la teleología de la decisión - errónea o acertada, es lo de menos-, al desvanecerse la transparencia del proceso desde el principio hasta el final, deviniendo opacos para sus creadores, incapaces de rastrear la lógica que siguen. De ahí que se hable del proceso de adopción de la decisión por parte de los algoritmos como una caja negra (black box).

matemático o informático (a pesar de que los algoritmos también se pueden expresar de otras muchas maneras, incluyendo los diagramas de flujo, el pseudocódigo y el propio lenguaje natural)". 


\section{Resignificación de las fuentes del derecho}

Desde un prisma estrictamente jurídico, mayor trascendencia que la definición del algoritmo, lo tiene la catalogación jurídica del mismo, ya sea identificándolo como fuente o no de derecho, para el caso o supuesto que ese algoritmo formara parte de la decisión jurisdiccional, como determinando si son actos administrativos, normas o si pueden integrarse en el concepto de actuación automatizada.

Considera De La Cueva ${ }^{4}$ que en la escritura del código fuente y de los algoritmos utilizados o que pudieran emplearse para los sistemas de gestión, de control o mutatis mutandi, en el ámbito de la decisión jurisdiccional, deberían exigirse los mismos requisitos que se establecen para las fuentes tradicionales del derecho, a saber: legalidad; regulación competencial; transparencia procedimental; publicidad normativa y naturaleza pública demanial.

Otros $^{5}$, sin embargo, consideran innecesaria esa previsión, desde la premisa de que la fuente de derecho no cambia, sigue siendo la norma jurídica - en las manifestaciones que prevé el art. 1.6. del Código Civil-, siendo la variable el vehículo de su ejecución o aplicación, en este caso, el algoritmo y el código fuente, lo cual, no obstante, «no supone en modo alguno que también sea preciso dotarles de un procedimiento debido administrativo para su adopción, del que se carece hasta el momento, de una motivación comprensible, cuya exigencia no existe, y de una transparencia adecuada, que incluya la posibilidad de participación ciudadana en su elaboración, su publicación y el derecho de acceso».

En cualquier caso, quizá lo más operativo en este ámbito sería delimitar las funciones y usos de la IA y los sistemas empleados (deterministas, predictivos, prescriptivos, con aprendizaje supervisado o no, etc.). Sólo a partir de un suficiente conocimiento técnico y funcional es posible discriminar el tipo e intensidad de las garantías jurídicas exigibles.

4 Código fuente, algoritmos y fuentes del derecho, El notario del siglo XXI: revista del Colegio Notarial de Madrid, núm. 77 (2018), pp. 24 a 27.

5 Ponce Sole. J: Inteligencia artificial, derecho administrativo y reserva de humanidad: algoritmos y procedimiento administrativo debido tecnológico, Revista general de derecho administrativo (lustel, enero 2019, n.ㅇ5). 


\section{Marco normativo}

Y es que, precisamente, de lo que adolece este escenario pretendidamente disruptivo es de un marco legal solvente que dispense seguridad jurídica tanto al emitente como al destinatario de la decisión generada por el software.

Más allá de los programas de investigación y desarrollo de naturaleza estratégica que únicamente están al alcance de megapotencias como la República Popular China y los Estados Unidos $^{6}$, en el ámbito de la Unión Europea, las primeras iniciativas sobre el particular pueden encontrarse en el Comité de Asuntos Legales del Parlamento Europeo, que fue comisionado para la elaboración de un estudio para evaluar y analizar, desde una perspectiva legal y ética, un determinado número de normas de derecho civil sobre robótica en el ámbito europeo.

Del mes de junio de 2016 es el Estudio del Parlamento Europeo sobre la ética de los sistemas ciberfísicos y cómo cambiarán los robots nuestras vidas realizado por el Grupo de Expertos de Evaluación de las Opciones Científicas y Tecnológicas (STOA) y dirigido por la Unidad de Prospectiva Científica, así como la Carta sobre robótica en la que se propone por vez primera un código de conducta ética para los ingenieros en robótica, un código deontológico para los comités de ética de la investigación, así como sendas licencias para diseñadores y usuarios.

Un año después, el Parlamento Europeo aprobó la Resolución del Parlamento Europeo, de 16 de febrero de 2017, con recomendaciones destinadas a la Comisión sobre normas de derecho civil sobre robótica (2015/2103(INL), la primera respuesta normativa positivizada a los retos jurídicos planteados por la automatización.

Mucha más relevancia, en lo que aquí interesa, tuvo, sin embargo, la Carta Europea sobre el uso ético de la inteligencia artificial en los sistemas judiciales y su entorno, aprobada en diciembre de 2018 por la Comisión Europea para la Eficiencia de la Justicia. En ella, se fijaron los cinco principios éticos

6 National IT Development Strategy (2016-2020) de la República Popular China y la The national artificial intelligence research and development strategic plan (2016) norteamericana. 
estructurales que deben informar el empleo de la IA por parte de un juzgado o tribunal, pero también aplicables a cualquier operador jurídico que trabaje en su entorno:

a. Principio de respeto de los derechos fundamentales, que garantice que el diseño e implementación de herramientas y servicios de IA son cohonestables con los derechos fundamentales, priorizándose el enfoque humanístico tanto en la fase de diseño como de aprendizaje.

b. Principio de no discriminación, en aras de prevenir el desarrollo hipertrófico de cualquier discriminación entre individuos 40 grupos de individuos, habida cuenta de la extraordinaria capacidad de estos sistemas para aflorar la discriminación existente cuando procesa, directa o indirectamente, información sensible relativa, verbi gratia, a opiniones políticas, datos genéticos o sobre la orientación sexual de cada individuo. El amenazador sesgo del que más adelante hablaremos desde un prisma aplicativo.

c. Principio de calidad y seguridad, que debe estar presente en todo momento durante el procesamiento de decisiones y datos judiciales, nutriéndose exclusivamente de fuentes certificadas y datos intangibles, con modelos elaborados de forma multidisciplinar en un entorno tecnológico seguro, permitiendo una completa trazabilidad durante todo el proceso que garantice la inmutabilidad del contenido o del significado de la decisión durante su procesamiento.

Es asimismo medular que los diseñadores de modelos de aprendizaje automático, al crear los algoritmos, tengan muy en cuenta el conocimiento y experiencia de profesionales del sistema judicial, más allá de su pericia técnica sectorial.

Finalmente, se impone la generalización de estudios de evaluación de riesgos e impactos ex ante, especialmente en relación con aquellas normas jurídicas que vayan a prever su aplicación mediante IA, las conocidas como machine-readable laws; particulares controles periódicos 
de calidad y auditorías, en su caso independientes, así como medidas específicas para la minimización de datos, anonimización y seudo anonimización.

Con respecto a esto último, es por todos sabido que el Tribunal Constitucional y el Tribunal Supremo mantienen dos enfoques bien distintos en lo que al tratamiento de datos en sus sentencias se refiere. Mientras que el máximo tribunal de garantías de España ${ }^{7}$ - como el Tribunal de Justicia de la Unión Europea (TJUE), el Tribunal Europeo de Derechos Humanos (TEDH), o las cortes supremas norteamericana y británica- incluye los nombres de los demandantes y demandados en sus resoluciones judiciales que, después, publica en su página web, el Tribunal Supremo -y el resto de órganos jurisdiccionales- lo somete a un proceso de anonimización a cargo del Centro de Documentación Judicial (CENDOJ ) del Consejo General del Poder Judicial (CGPJ) en el que se expurgan nombres, direcciones y otros datos personales de las partes, con el objeto de asegurar el cumplimiento de los fines encomendados al referido centro por el Reglamento 1/1997, del CGPJ, aprobado por Acuerdo de 7 de mayo de 1997, procurando

7 “En primer lugar, ha de incidirse en que la Ley Orgánica de este tribunal establece, por un lado, y en su artículo 1.1, que "[e]l Tribunal Constitucional, como intérprete supremo de la Constitución, es independiente de los demás órganos constitucionales y está sometido solo a la Constitución y a la presente Ley Orgánica", dice la mencionada sentencia.

“En cuanto a la publicidad y publicación de las resoluciones jurisdiccionales del Tribunal Constitucional, las previsiones establecidas en la Constitución son, por un lado, el artículo 120 de la Constitución española, en cuyos apartados primero y tercero se establece, respectivamente, el principio general de que 'las actuaciones judiciales serán públicas, con las excepciones que prevean las leyes de procedimiento", y que "las sentencias serán siempre motivadas»

«Por otro, y muy especialmente, el art. 164.1 CE que establece que 'las sentencias del Tribunal Constitucional se publicarán en el boletín oficial del Estado con los votos particulares, si los hubiere', incidiendo, además, en que el valor de cosa juzgada la adquieren a partir del día siguiente de su publicación.

Por su parte, las previsiones establecidas en la Ley Orgánica del Tribunal Constitucional sobre el particular son, por un lado, el art. 86.2, concretando que la obligación de publicación en el Boletín Oficial aparece referida tanto a las sentencias como a las declaraciones sobre la constitucionalidad de los tratados internacionales y que la misma debe producirse dentro de los 30 días siguientes a la fecha del fallo; y, por otro, el art. 99.2, que establece, como obligación del Tribunal Constitucional 'la recopilación, clasificación y publicación de la doctrina constitucional del Tribunal'". STC 114/2006 de 5 de abril de 2006 del Tribunal Constitucional. 
la supresión de los datos de identificación para asegurar en todo momento la protección del honor e intimidad personal y familiar (art.5.3 bis).

d. Principio de transparencia, imparcialidad y equidad, lo cual exige que la utilización de herramientas que pueden tener consecuencias o afectar significativamente la vida de las personas, venga regida por un equilibrio entre la propiedad intelectual de ciertos métodos de procesamiento y la necesidad de transparencia, lo que ciertamente resulta una tarea complicada dado el impacto económico que actualmente representa el sector de las legaltech.

Desde otro punto de vista, es perentoria la reestructuración organizativa de la administración a través de nuevas unidades orgánicas y dinámicas que combinen el conocimiento técnico y jurídico bajo los principios de legalidad, eficiencia y coordinación entre los diferentes niveles administrativos, asesoradas por comisiones éticas para la adecuada incorporación de la IA, todo ello complementado con la articulación de departamentos técnicos que diseñen sistemas automatizados especialmente enfocados para el sector público y de un marco contractual que facilite la gestión frente a grandes prestadores de servicios. Todo ello implica, consecuentemente, una nueva política de empleo público, en la que se pondere la presencia de un perfil más técnico y mejor cualificado del servidor público.

e. Y, finalmente, el principio de la autonomía del usuario, tendente a potenciar y no a restringir el control que sobre la decisión generada por el software debe disponer el usuario. Así, los profesionales del sistema de justicia deben, en cualquier momento, ser capaces de revisar las decisiones judiciales y los datos utilizados para producir un resultado, evitando la vinculación irreversible con la decisión generada por la IA a la luz de las características específicas del caso particular.

Una delimitación de espacios de reserva de humanidad, vedados por tanto a las decisiones automatizadas, que tanto en su vertiente argumentativa como en la preservación del 
principio de legalidad y de reserva de ley, deben garantizar al legislador humano el control de la legitimación de tratamientos de datos por uso público de IA, así como, especialmente, al perfilar las garantías básicas en la línea de la exigente y consolidada jurisprudencia creada.

\section{Positivación normativa}

Descendiendo ya a la regulación positiva, en el ámbito comunitario, la disposición referencial es el Reglamento (UE) 2016/679 del Parlamento Europeo y del Consejo del 27 de abril de 2016 relativo a la protección de las personas físicas en lo que respecta al tratamiento de datos personales y a la libre circulación de estos datos y por el que se deroga la directiva 95/46/CE (Reglamento general de protección de datos) (texto pertinente a efectos del EEE). Esta disposición contempla algunos aspectos interesantes relacionados con la IA en la toma de decisiones administrativas, que no jurisdiccionales, como los contemplados en sus artículos 13 (información que deberá facilitarse cuando los datos personales se obtengan del interesado), 14 (información que deberá facilitarse cuando los datos personales no se hayan obtenido del interesado) y 15 (derecho de acceso del interesado) ${ }^{8}$.

8 "La existencia de decisiones automatizas, incluida la elaboración de perfiles, a que se refiere el artículo 22, apartados 1 y 4 , y, al menos en tales casos, información significativa sobre la lógica aplicada, así como la importancia y las consecuencias previstas de dicho tratamiento para el interesado"

Las «decisiones automatizadas» que referencia en el artículo 22 son:

Decisiones individuales automatizadas, incluida la elaboración de perfiles

1. Todo interesado tendrá derecho a no ser objeto de una decisión basada únicamente en el tratamiento automatizado, incluida la elaboración de perfiles, que produzca efectos jurídicos en él o le afecte significativamente de modo similar.

2. El apartado 1 no se aplicará si la decisión:

a. Es necesaria para la celebración o la ejecución de un contrato entre el interesado y un responsable del tratamiento.

b. Está autorizada por el Derecho de la Unión o de los Estados miembros que se aplique. Al responsable del tratamiento y que establezca asimismo medidas adecuadas para salvaguardar los derechos y libertades y los intereses legítimos del interesado.

c. Se basa en el consentimiento explícito del interesado.

3. En los casos a que se refiere el apartado 2, letras a. y c., el responsable del tratamiento adoptará las medidas adecuadas para salvaguardar los derechos y libertades y los intereses legítimos del interesado, como mínimo el derecho a obtener intervención humana por parte del responsable, a expresar su punto de vista y a impugnar la decisión. 
El ordenamiento jurídico español carece hoy, tanto de una planificación de políticas públicas como de una producción legislativa digna de ese nombre respecto a la regulación de las tecnologías autónomas, más allá de la relacionada con la progresiva y lenta implantación de las Tecnologías de la Información y la Comunicación (TIC) a las administraciones públicas.

Desde una perspectiva puramente normativa, debe citarse, naturalmente, la Ley Orgánica 3/2018, de 5 de diciembre, de protección de datos personales y garantía de los derechos digitales que, sobre este particular, en su artículo 11, al tratar la transparencia e información al afectado, dispone que, si los datos obtenidos del afectado fueran a ser tratados para la elaboración de perfiles, la información básica comprenderá asimismo esta circunstancia. En este caso, el afectado deberá ser informado de su derecho a oponerse a la adopción de decisiones individuales automatizadas que produzcan efectos jurídicos sobre él o le afecten significativamente de modo similar, cuando concurra este derecho de acuerdo con lo previsto en el artículo 22 del Reglamento (UE) 2016/679.

Referencias más específicas a la actividad administrativa automatizada pueden encontrarse en la Ley 58/2003, de 17 de diciembre, General Tributaria, que en su artículo 96 - bajo el lema de la "utilización de tecnologías informáticas y telemáticas"- se refiere al empleo por parte de la administración tributaria de las nuevas tecnologías en el desarrollo de su actividad ${ }^{9}$.

4. Las decisiones a que se refiere el apartado 2 no se basarán en las categorías especiales de datos personales contempladas en el artículo 9, apartado 1, salvo que se aplique el artículo 9, apartado 2, letra a. o g., y se hayan tomado medidas adecuadas para salvaguardar los derechos y libertades y los intereses legítimos del interesado".

9 “Artículo 96. Utilización de tecnologías informáticas y telemáticas.

1. La Administración tributaria promoverá la utilización de las técnicas y medios electrónicos, informáticos y telemáticos necesarios para el desarrollo de su actividad y el ejercicio de sus competencias, con las limitaciones que la Constitución y las leyes establezcan.

2. Cuando sea compatible con los medios técnicos de que disponga la Administración tributaria, los ciudadanos podrán relacionarse con ella para ejercer sus derechos y cumplir con sus obligaciones a través de técnicas y medios electrónicos, informáticos o telemáticos con las garantías y requisitos previstos en cada procedimiento.

3. Los procedimientos y actuaciones en los que se utilicen técnicas y medios electrónicos, informáticos y telemáticos garantizarán la identificación de la Administración tributaria actuante y el ejercicio de su competencia. Además, cuando la Administración 
Asimismo, la Ley 11/2007, de 22 de junio, de acceso electrónico de los ciudadanos a los servicios públicos, además de algunas breves menciones en conexión con los sistemas de firma electrónica, se ocupa en sus artículos 38 y 39 y en su anexo de la actividad administrativa automatizada.

Además de esta regulación hay que tener en cuenta las referencias a la actuación administrativa automatizada y sus sistemas de firma que contemplan epidérmicamente los arts. 40 y 41 de la Ley 40/2015, de 1 de octubre, de Régimen Jurídico del Sector Público.

En el sector de la Administración de Justicia, el acentuado atraso de la misma, en plena transición hacia el expediente electrónico y la interoperabilidad entre todas las aplicaciones informáticas en uso, atomizadas en una pluralidad de territorios y colectivos, augura particularmente lejana la llegada de las tecnologías que estamos examinando.

La Ley Orgánica 16/1994 reformó la Ley Orgánica del Poder Judicial de 1 de julio 1985 en su artículo 230 para habilitar jurídicamente por primera vez medios técnicos, electrónicos, informáticos y telemáticos puestos a su disposición, de modo obligatorio y con pleno valor legal, en una redacción que fue reforzada posteriormente por la Ley Orgánica 7/2015.

Finalmente, la Ley 18/2011 reguladora del uso de las tecnologías de la información y la comunicación en la Administración de Justicia, se limita a establecer con cierto detalle la habilitación normativa necesaria para la transformación de la Administración de Justicia en una Administración electrónica.

tributaria actúe de forma automatizada se garantizará la identificación de los órganos competentes para la programación y supervisión del sistema de información y de los órganos competentes para resolver los recursos que puedan interponerse.

Los programas y aplicaciones electrónicos, informáticos y telemáticos que vayan a ser utilizados por la administración tributaria para el ejercicio de sus potestades habrán de ser previamente aprobados por esta en la forma que se determine reglamentariamente.

Los documentos emitidos, cualquiera que sea su soporte, por medios electrónicos, informáticos o telemáticos por la administración tributaria, o los que esta emita como copias de originales almacenados por estos mismos medios, así como las imágenes electrónicas de los documentos originales o sus copias, tendrán la misma validez y eficacia que los documentos originales, siempre que quede garantizada su autenticidad, integridad y conservación y, en su caso, la recepción por el interesado, así como el cumplimiento de las garantías y requisitos exigidos por la normativa aplicable." 


\section{Viabilidad judicial}

Sabemos ya lo que es un algoritmo y su evolución tecnológica. También nos hemos planteado las exigencias que una calificación como fuente de derecho, en su caso, supondría. Conocemos el escenario normativo que opera sobre la cuestión, especialmente magro en el sector de la justicia. Es hora ya de abordar el tuétano de estas reflexiones, que no es otro que delimitar el verdadero efecto disruptivo de la IA en la decisión judicial. Para ello, se impone, en primer término, sondear cómo a nivel internacional se contempla este escenario para, después, compararlo con el estado de la cuestión en nuestro sistema judicial.

Como premisa altamente indicativa del potencial desarrollo y real incidencia de esta mecánica, debe señalarse que The Partnership on $\mathrm{Al}$, una asociación que reúne a gigantes digitales como Apple, Amazon, Facebook, IBM, Nvidia, Microsoft y Google, se han posicionado claramente en contra de emplear la inteligencia artificial para dictar sentencias en asuntos de naturaleza penal.

Una de las barreras que apunta el consorcio de las grandes tecnológicas de Silicon Valley es el sesgo a la hora de aplicar el derecho. «El método correcto que se empleará dependerá de lo que signifique que una herramienta sea justa en una aplicación en particular, por lo que esta no es solo una cuestión técnica, sino también una cuestión de ley, política y ética», apuntan. «Si bien no existe una solución única para abordar el sesgo, a continuación, se presentan algunos de los posibles enfoques que podrían ser apropiados en el contexto de las predicciones de evaluación de riesgo de Estados Unidos», añade.

Con similar prudencia también se expresa el Dr. Nikolaos Aletras, director de un proyecto auspiciado por el University College of London, Sheffield y Pennsylvania y la compañía Amazon Predicting judicial decisions of the European Court of Human Rights: a Natural Language Processing perspective (2016) conforme al cual se han aplicado los recientes avances tecnológicos en aprendizaje automático (machine learning) y procesamiento del lenguaje natural (NPL) para construir un modelo predictivo que permitiera identificar patrones en la actividad resolutiva de un órgano judicial. En esta ocasión se trató del 
primer estudio sistemático para la predicción del sentido básico en que se resuelven los asuntos del TEDH. Tras examinar 584 casos materialmente relacionados con los artículos 3, 6 y 8 de la Convención Europea de Derechos Humanos, y procesalmente limitados a casos ingresados y admitidos a trámite, el modelo consiguió predecir el sentido del fallo con un porcentaje de acierto del $79 \%$, lo que es, sin duda, una cifra significativa.

Dos años antes, en el estudio A General approach for predicting the behaviour of the Supreme Court of the United States (2014) llevado a cabo por investigadores de Illinois Tech-Chicago Kent College of Law, LexPredict, LLC and South Texas College of Law Houston, se analizaron los resultados de la implementación de un algoritmo que consideraba más de 90 variables y que examinaba 60 años de datos del Tribunal Supremo de los Estados Unidos, 7700 asuntos y más de 68000 votos particulares, obteniendo un porcentaje de acierto del $70 \%$ en el sentido de las resoluciones y del $71 \%$ en votos particulares de los magistrados.

Pues bien, a pesar de las precauciones advertidas por los desarrolladores y programadores de IA, estos sistemas se están usando en la actualidad. Estados Unidos trabaja ya con el Correctional Offender Management Profiling for Alternative Sanctions [COMPAS], un algoritmo diseñado para, a partir del manejo de extensas bases de datos y de respuestas a cuestiones formuladas a un presunto infractor, predecir el riesgo de comisión de nuevas infracciones legales, asistiendo y sirviendo de apoyo al juez para la toma de una decisión.

Asimismo, en la República Popular China, en la provincia de Hebei (con una extensión de $190000 \mathrm{~km}^{2}$ y una población de 65 millones de habitantes) 178 órganos judiciales locales utilizan desde julio de 2016 la aplicación Intelligent trial 1.0, un sistema de asistencia virtual basado en el manejo de vastos caladeros de datos, cuyos resultados han ayudado a cerca de 3000 jueces en más de 150000 asuntos, reduciendo en una tercera parte su carga de trabajo.

En Europa, el país más avanzado en estas dinámicas es, sin lugar a dudas, Estonia, poniéndose a la cabeza de la investigación de los vulgarmente denominados «jueces robots» para la resolución de demandas de cantidad hasta un máximo de 7000 euros. 
No obstante todo lo anterior, también hay comunidades jurídicas que empiezan a plantear limitaciones legales al uso indiscriminado de las Legaltech. Francia, en su nueva Ley para la reforma de la Justicia, establece penas de cárcel de hasta cinco años para quien publique información estadística sobre las decisiones y el patrón de conducta de los jueces en cuanto a las sentencias que dictan ${ }^{10}$. Una prohibición que, de facto y de iure, limita parte de la funcionalidad de herramientas Legaltech como la francesa Prédictice, pero también, en caso de que hubiere una legislación análoga, a la norteamericana Context de Lexis Nexis o la española Jurimetríca.

\section{El asesoramiento jurídico privado}

Es indudable que los mayores avances en las llamadas tecnologías disruptivas en el ámbito legal están claramente relacionados con la actividad del asesoramiento jurídico privado. Las grandes firmas y despachos de abogados están realizando importantes inversiones en la automatización de tareas dirigida tanto al ahorro de recursos personales como, simultáneamente, a la racionalización y mejora de la calidad de los servicios que prestan: sistemas de procesamiento de grandes volúmenes de legislación y jurisprudencia para ofrecer respuestas selectivas; análisis simultáneo de millones de litigios; revisión de contratos o verificación de brechas de seguridad; conversión de expresiones legales en mapas de palabras entendibles por cada uno de los actores que intervienen en la elaboración de los documentos legales; sugerencias de cláusulas al tiempo de la redacción de documentos; almacenamiento de contratos elaborados en diferentes despachos para trabajar sobre ellos en la redacción de los nuevos o software de reconocimiento de voz que evita que los letrados tengan que redactar miles de documentos en litigaciones.

10 El artículo 33 de esa ley establece concretamente (traducción desde la versión original en francés): los datos de identidad de los magistrados y miembros del registro no pueden ser reutilizados con el propósito o el efecto de evaluar, analizar, comparar o predecir sus prácticas profesionales reales o presuntas. La violación de esta prohibición se castiga con las sanciones previstas en los artículos 226-18,226-24 y 226-31 del Código Penal, sin perjuicio de las medidas y sanciones previstas en la Ley № 78-17, de 6 de enero de 1978, en relación con el procesamiento de datos, archivos y libertades." 
Según los datos extraídos del mapa Legaltech de la consultora Legaltechies, en junio de 2019 había 142 proyectos y más de un centenar de empresas relacionadas con este sector a lo largo y ancho de todo el territorio español.

En el ámbito de la resolución alternativa de conflictos, también la mediación experimenta el impacto de la introducción de las TIC, señaladamente en el ámbito civil y mercantil, en el contexto del imparable avance del comercio electrónico. Como consecuencia de la Directiva 2013/11/UE del Parlamento Europeo y del Consejo, de 21 de mayo de 2013 sobre resolución alternativa de litigios en materia de consumo, y del reglamento (UE) 524/2013 del Parlamento Europeo y del Consejo, de 21 de mayo de 2013, sobre resolución de litigios en línea en materia de consumo, la comisión europea puso en funcionamiento en enero de 2016 una plataforma electrónica para la resolución alternativa de conflictos en materia de consumo. Repárese en que no se trata de un sistema automatizado, sino de una vía de comunicación privilegiada entre la empresa y el consumidor. Como experiencias en funcionamiento aplicadas a conflictos de distinta naturaleza (por ejemplo, de derecho de familia o propiedad inmobiliaria) se puede citar también el Proyecto Rechtwijzer en los Países Bajos, fruto de la colaboración entre el Instituto de La Haya para la internacionalización de la ley (HIIL), el Consejo Holandés de Asistencia Jurídica y la firma estadounidense Modria.

\section{La realidad y el deseo: I A y decisión judicial}

Después de este breve itinerario digital ¿alguna conclusión? Sí, que la sustitución directa de la actividad humana en la decisión judicial, hoy en día, es puramente quimérica a corto y medio plazo. Otra cosa son las actividades predictivas al socaire de las plataformas privadas que emplean la IA que, como se ha visto, están plenamente desarrolladas $y$, en algunos casos, como en Francia, tipificadas penalmente para determinados supuestos. Junto a esta actividad comercial presciente, únicamente en dos ámbitos puede afirmarse con rigor una tímida disrupción en el ámbito judicial: las posibilidades que ofrece la automatización simple en el proceso y el empleo de tecnologías de procesamiento automático de datos en el ámbito audiovisual. 
Con respecto al primer aspecto, recordemos que el artículo 237 LOPJ establece, en su redacción dada por Ley Orgánica $19 / 2003$, que salvo que las leyes procesales dispongan otra cosa, se dará de oficio al proceso el impulso que corresponda, dictándose al efecto las disposiciones necesarias. El artículo 456 LOPJ señala que corresponde a los Letrados de la Administración de Justicia (LAJ) la función de impulso del proceso, mediante las correspondientes diligencias.

La Ley de Enjuiciamiento Civil de 1881 (LEC) establecía el principio de impulso de parte, es decir, se iría pasando de un trámite procesal a otro o de una fase procesal a otra en la medida en que las partes activaran el curso del proceso e indicaran que se transitará a la siguiente actuación. La consecuencia era que, en caso de no producirse dicha activación del curso procesal, se producía la caducidad de la instancia, como modo de terminación del proceso. Esta situación cambiaría con la reforma de la Ley 34/1984, que estableció finalmente el impulso de oficio en los procedimientos civiles y, consecuentemente, la preclusión en el caso de que la parte procesal no verificase el trámite correspondiente dentro de plazo y el consiguiente pase a la fase siguiente una vez finalizado el plazo correspondiente.

En la actualidad, el artículo 236 LEC contempla específicamente el impulso de oficio, al señalar que la falta de impulso del procedimiento por las partes o interesados no originará la caducidad de la instancia ni del recurso, si bien, añade el artículo 237 LEC, se entenderán por abandonadas las instancias y recursos en todo tipo de pleitos si, pese al impulso de oficio de las actuaciones, no se produce actividad procesal alguna en el plazo de dos años, si el pleito se hallara en primera instancia y un año si se hallara en segunda instancia; y con independencia de si estuviera pendiente la casación o recurso extraordinario por infracción procesal. Y ello, no obstante, añade el artículo 238 LEC, en caso de fuerza mayor o cuando la inactividad procesal se debiera a causas ajenas a la voluntad de las partes o de los interesados, no se producirá la caducidad de la instancia, que tampoco se aplicará en el caso de las actuaciones de ejecución forzosa, conforme al artículo 239 LEC. Los efectos de la caducidad de la instancia, según el artículo 240 LEC, son diferentes según estemos ante la primera instancia o ante un recurso; en el primer caso el efecto jurídico es equivalente a un desistimiento y por tanto se entiende sin perjuicio de la presentación de nueva demanda; 
pero si la caducidad de la instancia se produce dentro de un recurso, entonces la sentencia habrá quedado firme en tanto en cuanto equivale dicha caducidad al desistimiento del recurso.

Correlativamente al impulso de oficio, la LEC dispone en su artículo 134 la improrrogabilidad de los plazos procesales, sin perjuicio de la interrupción de plazos o demora de términos en caso de fuerza mayor que impida el cumplimiento de aquellos; y sin perjuicio de la regla sobre presentación de escritos de término que establece el artículo 135 LEC. Y, en consecuencia, el artículo 136 LEC señala que, transcurrido el plazo o término señalado para la realización de una actividad procesal por la parte, se producirá la preclusión y se perderá la oportunidad de realizar el acto de que se trate.

Pues bien, este tradicional régimen de impulso procesal puede tener las horas contadas. $Y$ es que algunas aplicaciones de gestión procesal como la que maneja e-justicia.cat ya prevén en su módulo de tramitación de expediente electrónico la generación automática del siguiente trámite legalmente previsto, en forma de sugerencia $\mathrm{y}$, por tanto, al margen de la iniciativa del LAJ. No es difícil ya contemplar, por tanto, una hipótesis próxima en la que determinados procedimientos y sus trámites procesales, - una vez valorada por la propia aplicación la adecuación de requisitos estrictamente formales-, sean impulsados de modo automatizado y notificados mediante la integración en plataformas de comunicación. Las posibilidades de automatización dependerán obviamente de la complejidad y exigencias de garantía de la regulación procesal afectada.

Con respecto al campo de la documentación audiovisual de actos procesales, las más avanzadas plataformas de reproducción de contenidos audiovisuales ya ofrecen posibilidades, como la transcripción o traducción simultáneas o posteriores a la grabación que, pese a estar en producción y uso en diferentes sectores, aún están lejos de ser implantadas en el sector público judicial. El estado actual de la tecnología ha superado la mera grabación de la imagen y el sonido, bien sea mediante técnicas de realidad aumentada, añadiendo elementos virtuales a la reproducción, bien mediante el procesamiento de datos obtenidos por la propia cámara que bien podrían ser aplicadas en la fase de prueba, evitando así innecesarios desplazamientos de la comisión judicial y engorrosos levantamientos de actas decimonónicas. 
Fuera de estos concretos supuestos, ha de insistirse, a fuer de ser tachado de aguafiestas digital, todo es ciencia ficción. Y dificultades. Ya se ha hecho referencia en páginas precedentes a la polémica del software COMPAS utilizado en los Estados Unidos para la predicción del riesgo de futuros delitos que ha sido seriamente puesta en duda por su sesgo racista, al atribuir un $77 \%$ más de riesgo delictivo a la población negra que a la blanca. En el año 2013, Eric Loomis fue detenido por agentes de policía del Estado de Wisconsin cuando conducía un vehículo implicado en un reciente tiroteo, por lo que fue acusado de huir de la policía y utilizar un vehículo sin la autorización de su propietario. El encartado se declaró culpable de ambos delitos con la esperanza de eludir el ingreso en prisión. Durante la vista para decidir sobre su libertad condicional, el fiscal aportó un informe elaborado por el programa informático COMPAS, según el cual, el acusado tenía un riesgo elevado de reincidencia. Partiendo de tales consideraciones, el juez le impuso una pena de seis años de prisión y otros cinco en régimen de libertad vigilada. La defensa del condenado recurrió la sentencia alegando que se había vulnerado el derecho a un proceso con todas las garantías porque no podía discutir los métodos utilizados por el programa informático COMPAS, dado que el algoritmo era secreto y solo lo conocía la empresa que lo había desarrollado. Sin embargo, tales argumentos no fueron acogidos por la Corte Suprema del Estado de Wisconsin, que consideró que el programa informático se había basado únicamente en los factores habituales para medir la peligrosidad criminal futura como, por ejemplo, huir de la policía y el historial delictivo previo.

La pura búsqueda de la imitación tecnológica más exacta posible de las pautas del comportamiento humano conduce al riesgo de incurrir en una reproducción no selectiva de las mismas. Por ello el consenso y la estandarización política de valores democráticos y éticos en los procesos de robotización debe ser una prioridad para los poderes públicos.

En segundo lugar, la posible falta de control político democrático sobre el desarrollo e implantación de las tecnologías que tiendan a suplir el factor humano en el ámbito de la decisión judicial, unida a la mitificación de una visión maximalista de la independencia judicial y su despolitización o desideologización, son elementos cuya interacción puede tener un impacto directo en la percepción que la sociedad debe tener de la administración 
de justicia. Repárese que la clave de bóveda de esa favorable percepción sociológica radica en la expectativa cultural de que los jueces deben comportarse independientemente. Ser un juez en los Estados Unidos es, en palabras del magistrado de su Tribunal Supremo Stephen Breyer «en parte un estado mental, una cuestión de expectativas, hábito y creencias, no sólo entre los jueces, abogados y legisladores, sino entre millones de personas». En la medida en que la sociedad, merced a medidas de transparencia y acceso a la información, se forma una opinión sobre la actividad de los tribunales, la confianza pública en el Poder Judicial se incrementa de manera exponencial. La razón más importante para confiar en que el fallo de un juez será eficaz, más allá de medidas coercitivas o garantías institucionales para su acatamiento, es de índole cultural y no institucional. La ciudadanía ha interiorizado el acatamiento natural de los fallos de los tribunales desde la premisa moral de que, si los casos son sustanciados por jueces imparciales, libres de influencia política, los justiciables se conducirán de acuerdo con la ley y con su interpretación judicial.

Estamos por tanto ante lo que se podría denominar un ejercicio de «legitimidad social», es decir, el grado de confianza y de credibilidad social que una institución (el sistema de Justicia en este caso) amerita entre la ciudadanía. Es este un rasgo imprescindible de los sistemas de Justicia, no un atributo opcional o contingente: de una justicia eficiente, pero sin autoridad moral difícilmente pueden esperarse frutos. El buen sistema de justicia debe generar en sus justiciables (reales o potenciales) el convencimiento de que sus asuntos van a ser tratados no solo diligente y competentemente sino además de la forma más ecuánime, recta y equilibrada posible. De esto último depende fundamentalmente su fiabilidad, es decir, su legitimidad social. Adviértase que, si no fuese así, resultaría injustificable la expropiación a los particulares de la tentación de hacer justicia por su cuenta, no en vano, la potestas del Poder Judicial es necesaria pero no suficiente para que el sistema se muestre como confiable y digno de respeto; para ello precisa además de auctoritas, es decir, de la capacidad moral para emitir una opinión cualificada sobre una decisión. El nivel de legitimidad social correspondiente a un sistema de justicia puede ser entendido como el residuo cristalizado de la forma en que la ciudadanía le percibe y evalúa en relación con rasgos básicos tales como su nivel de independencia e imparcialidad y, sobre todo, su grado de accesibilidad para el 
usuario, el trato que le dispensa y su margen de diafanidad a la hora de dar cuenta de su funcionamiento de conjunto, sin que además, este rasgo institucional objetivo pueda establecerse o imponerse legislativamente. Dejar en manos de un software esta frágil y umbilical relación exige un extraordinario acto de fe en la eficacia de este tipo de tecnología.

Por otra parte, la actual (in)capacidad de procesamiento de lenguaje humano por parte de los algoritmos actuales, afecta decisivamente a las posibilidades de interacción entre la persona y los sistemas artificiales, incapaces de sintetizar y asimilar las lagunas jurídicas, contradicciones, expresiones ambiguas, polisemias, homonimias o los conceptos jurídicos indeterminados propios de un lenguaje jurídico, por otra parte, profundamente politizado en su naturaleza y por tanto, aún menos aprehensible para el sistema de automatización.

En este sentido, resulta especialmente paradójico advertir cómo la febril búsqueda de una respuesta humana homogeneizada por medio de los procesos de síntesis del lenguaje humano, choca radicalmente con la tendencia advertible en los modelos procesales de las últimas instancias judiciales, en cuyas fases de admisión, es cada vez más acentuada la aplicación de criterios puramente discrecionales y, por ende, de muy difícil digestión por el algoritmo.

No puede tampoco dejarse a humo de pajas el impacto de una eventual adopción de políticas de automatización en la organización de la Administración de Justicia. Según un estudio referido al Reino Unido de comienzos de 2017, al menos 250.000 puestos de trabajo en el sector público serán reemplazados en su actividad por los procesos de robotización y automatización de tareas administrativas en los próximos quince años.

En la administración de justicia española es evidente el peso del factor humano en la administración de justicia. Para hacernos una idea de la importancia de los recursos personales en las políticas públicas de justicia basta con medir el capítulo de gastos de los Presupuestos Generales del Estado para la administración de justicia española, que en el ejercicio 2017 fue de 1726 millones de euros, de los cuales 1358 millones de euros fueron para gastos de personal y solo 368 millones para el resto de políticas públicas. 
En el momento actual no se ha registrado ni está prevista una sustitución tecnológica directa de puestos de trabajo, pero sí ha existido un limitado impacto organizativo, relacionado con la implantación combinada -aunque aún escasa y parcial- de la oficina judicial. No es aventurado pronosticar que la llegada de la segunda generación de sistemas informáticos de gestión procesal, la necesaria e inevitable integración de los mismos con otras aplicaciones y la implantación definitiva del anhelado expediente electrónico sólo pueden tener como siguiente paso una limitada irrupción de tecnologías disruptivas basadas en la automatización, y ello sí que puede tener un impacto directo y significativo en la configuración y organización de las plantillas humanas de la administración de justicia.

Y finalmente, nos topamos, como diría Graham Green, con el factor humano. Fíjense, por ejemplo, en un cambio normativo como el operado en la casación contenciosa por la LO 7/2015, importante, qué duda cabe, pero, insisto, no otra cosa que una nueva reforma procesal. Pues bien, ese cambio legislativo ha supuesto para el juez casacional un extraordinario esfuerzo a la hora de reeducar su posicionamiento frente al recurso, en el cual ya no debe buscar únicamente el ius litigatoris del recurrente sino su ius constituionis y la proyección de su relevancia hacia la comunidad jurídica. Imagínese ahora el impacto que una disrupción no ya sustitutiva, sino meramente asistencial, supondría tanto para el jurista tradicional despojado de su aura exegética, para convertirse en un mero operario cualificado de aplicaciones tecnológicas.

\section{La verdadera disrupción}

Supremacía cuántica. No, no me estoy refiriendo a conflictos convivenciales de naturaleza territorial, ni al apartheid sudafricano ni tampoco de la lucha por los derechos humanos al sur de la línea Mason-Dixon de los años cincuenta. Hablo de la información publicada en el pasado número 574 [págs. 505-510(2019)] de la revista Nature, en el que se podía leer la versión definitiva del artículo científico en el que la empresa Google explica el presunto mayor avance de la historia en computación cuántica, sosteniendo en ese artículo haber logrado la abracadabrante proeza de emplear tres minutos y veinte segundos para resolver una operación de cálculo de números aleatorios que al ordenador 
convencional más potente de la actualidad le hubiera llevado miles de años: en otras palabras, se habría alcanzado la referida «supremacía cuántica».

La ininteligibilidad de la mecánica cuántica y de sus inextricables contradicciones residen en la doble realidad que únicamente se advierte a nivel microscópico, dada la ambivalencia de los electrones, que son onda y corpúsculo a la vez. Lo realmente sensacional es que, mientras no miremos y exijamos a la partícula que se defina, ambas posiciones físicas conviven, manteniéndose así esa peculiar y extraordinaria coherencia cuántica, que confirma que la realidad sólo es interacción. La computación clásica opera, por el contrario, de forma binaria y no admite indefiniciones, o son «unos» o son «ceros», mientras que la mecánica cuántica se aprovecha de la superposición de aquellos valores binarios para afrontar problemas - hasta ahora- irresolubles.

Pues bien, no hay disciplina técnica o intelectual que se asemeje más al experimento de Schrödinger que el Derecho y, más específicamente, su aplicación por los tribunales de justicia. Si recuerdan el experimento concebido en 1935 por el físico vienés, mientras la caja permanecía cerrada, y la partícula de la que dependía la activación de la trampa mortal permanecía no develada, el gato que había en su interior estaba vivo y muerto a la vez, extremo que se simplificaba al abrirse la caja y activarse la partícula felicida, momento en el que el minino estaría vivo o muerto, de manera excluyente.

Pues bien, en escenarios litigiosos, y partiendo de una premisa fáctica incontrovertida y pacífica, la pretensión del recurrente, demandante o denunciante y la oposición de la contraparte, ontológicamente contradictorias, coexisten de forma simultánea, «siendo» ambas al mismo tiempo, en tanto en cuanto la firmeza de la sentencia no disuelva esa (in)coherencia interna procesal.

Y es que el derecho, como el más y mejor acabado mecanismo autocompositivo diseñado por el ser humano, es muy anterior al cientificismo de la Ilustración, que garantizaba que cualquier problema era susceptible de resolución aplicando el razonamiento lógico en un número finito de pasos. El derecho, como la física cuántica, se radica, por el contrario, en el terreno que Lewis Carroll situaba al otro lado del espejo, donde rige una 
lógica y una vinculación causa-efecto sensiblemente diferente. Alicia contemplaba el mundo de los adultos no como la «realidad», sino como una inversión de aquella al socaire de dos términos absolutamente definitorios en el idioma inglés: el common sense victoriano y su opuesto, el nonsense, como respuesta al convencionalismo arbitrario de unas reglas que, a los ojos de Alicia, carecían de sentido. No busquen justicia en el maravilloso país de los Tribunales, en todo caso, y no siempre, motivada, proporcionada y contextualizada aplicación de la ley. Y es que, créanme, en el actual contexto de posmodernidad hipertecnificada, no hay aún nada más verdaderamente disruptivo que el propio derecho.

\section{Bibliografía}

Aletras, n., Tsarapatsanis, D., Preoţiuc-Pietro, D., Lampos, V. Predicting Judicial Decisions of the European Court of Human Rights: A Natural Language Processing Perspective. Peer J Computer Science. 2: e93; DOI 10.7717/peerj-cs.93 (2016).

Arute, F. et alii: Quantum supremacy using a programmable superconducting processor. Nature no 574 [págs. 505-510(2019)].

Barrio Andrés, M. Ciberderecho Bases estructurales, modelos de regulación e instituciones de gobernanza de Internet. Tirant lo Blanch, Valencia, 2018.

Derecho Público e Internet: la actividad administrativa de regulación de la Red. Instituto Nacional de Administración Pública INAP, Madrid, 2017.

Legal Tech. La transformación digital de la abogacía. Wolters Kluwer, Madrid, 2019.

De la Cueva, J.: Código fuente, algoritmos y fuentes del Derecho, El notario del siglo XXI: Revista del Colegio Notarial de Madrid, núm. 77 (2018), págs. 24 a 27.

García de Enterría, E. La lengua de los derechos. La formación del Derecho público europeo tras la Revolución Francesa, Civitas, Madrid, 2009.

Ponce Sole. J: Inteligencia artificial, Derecho administrativo y reserva de humanidad: algoritmos y procedimiento administrativo debido tecnológico. Revista General de Derecho Administrativo (I ustel, enero 2019, no 50).

Turner., J.: Robot Rules. Regulating Artificial Intelligence. Palgrave McMillian, Cham, 2019. 